\title{
Mahgoub transform of Boehmians
}

\author{
A.M. Mahajan ${ }^{1}$ and M.S. Chaudhary ${ }^{2, *}$
}

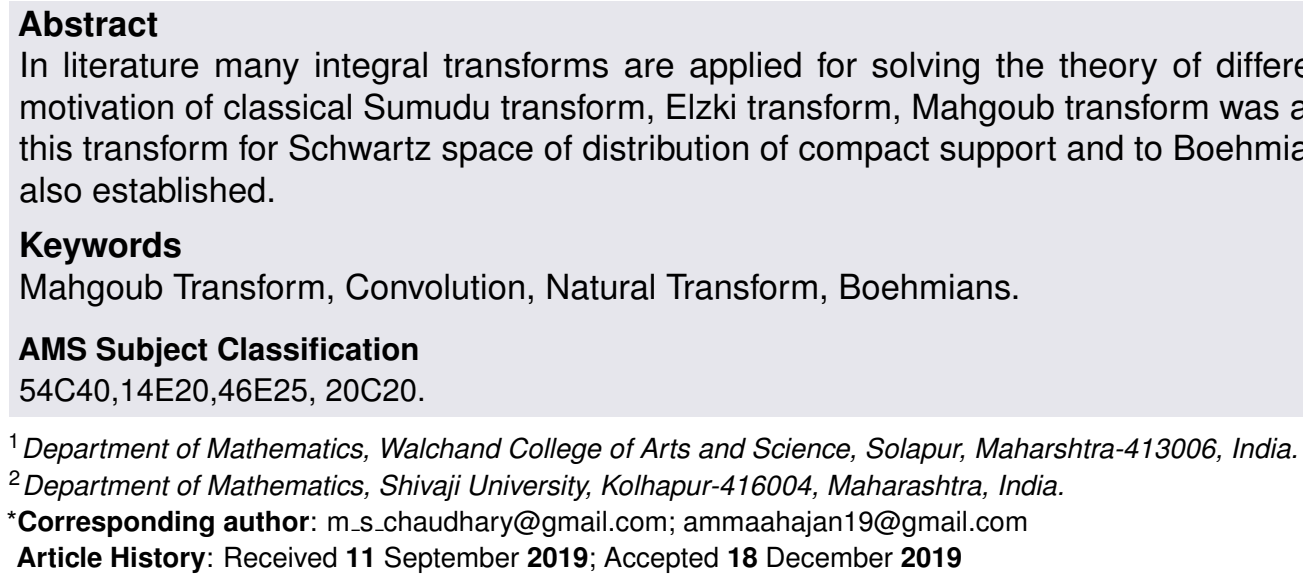
also established.

Keywords

Mahgoub Transform, Convolution, Natural Transform, Boehmians.

\section{AMS Subject Classification}

54C40,14E20,46E25, 20C20.

${ }^{1}$ Department of Mathematics, Walchand College of Arts and Science, Solapur, Maharshtra-413006, India.

${ }^{2}$ Department of Mathematics, Shivaji University, Kolhapur-416004, Maharashtra, India.

*Corresponding author: m_s_chaudhary@gmail.com; ammaahajan19@gmail.com

Article History: Received 11 September 2019; Accepted 18 December 2019 motivation of classical Sumudu transform, Elzki transform, Mahgoub transform was also developed. We extend this transform for Schwartz space of distribution of compact support and to Boehmian space. More results are

(C)2020 MJM.

\section{Contents}

1 Introduction $\ldots \ldots \ldots \ldots \ldots \ldots \ldots \ldots \ldots \ldots \ldots \ldots, 67$

2 Preliminaries 67

3 Relation between Mahgoub transform and other integral transforms .........................68

4 Mahgoub Transform of Distribution...........69

5 Mahgoub transform of Boehmians............70

6 Conclusion ............................ 72

References ........................... 72

\section{Introduction}

Mohand M.A. Mahgoub [12] introduced a new transform called Mahgoub transform. It is derived from classical Fourier integral. The Mahgoub transform has a strong relation with other integral transform. This relation is given by [9]. Mahgoub transform is used to facilitate the process of solving ordinary and partial differential equation in the time domain. From the motivation of Natural transform [10] the Mahgoub transform of a function $f(x)$ over the set where the set $\mathscr{A}$ is

$$
\mathscr{A}=\left\{f(x) / \exists K, J_{1}, J_{2}>0|f(x)|<K e^{|x| / J_{i}}\right\}
$$

for the given function in the set $\mathscr{A}$. The constant $\mathrm{K}$ must be finite. $J_{1}, J_{2}$ may be finite or infinite.

$$
M[f(x)]=\hat{M}(v)=v \int_{0}^{\infty} f(x) e^{-v x} \mathrm{~d} x \quad x \geq 0, \quad J_{1} \leq v \leq J_{2}
$$

Taha [9] gave the dualities between Mahgoub transform and other integral transform Sudhanshu Aggarwal [1, 3] gave the Solution of Linear volterra Integro Differential Equation of Second kind as well as the application for solving population growth and decay problems using Mahgoub transform, Aggarwal [2] also discussed the Linear ordinary differential equation using Mahgoub transform. S.K.Q. Al-omari [4] discussed the application of Natural transform and extended the Natural transform to generalized functions and Boehmians. Also, S.K.Q. Al-omari [5-7] gave the Hartly transform for $L^{p}$ Boehmians, Boehmians for optical fresnal warelet transform \& generalized Hartly Hilbert and Fourier Hilbert transform. A. H. Zemanian \& R.S. Pathak[13, 14] gave applications of generalized integral transforms in detail. In this paper we give the properties of classical Mahgoub transform, distributional Mahgoub transform and it's extension to Boehmian space.

\section{Preliminaries}

1. The Mahgoub transform of a constant function i.e. $f(t)=$ $\alpha$ is

$$
M(\alpha)=v \int_{0}^{\infty} \alpha \cdot e^{-v t} \mathrm{~d} t=\alpha
$$


2. The Mahgoub transform of $\mathrm{f}(\mathrm{t})=1$ is

$$
M(1)=v \int_{0}^{\infty} 1 \cdot e^{-v t} \mathrm{~d} t=1
$$

3. The Mahgoub transform of $\mathrm{f}(\mathrm{t})=\mathrm{t}$ is

$$
M(t)=\frac{1}{v}
$$

4. The Mahgoub transform of $\mathrm{f}(\mathrm{t})=e^{a t}$ is

$$
M\left(e^{a t}\right)=\frac{v}{v-a}
$$

5. The Mahgoub transform of $\mathrm{f}(\mathrm{t})=\sin a t, f(t)=\cos a t$ is

$$
\begin{aligned}
& M(\sin a t)=\frac{a v}{v^{2}+a^{2}} \\
& M(\cos a t)=\frac{v^{2}}{v^{2}+a^{2}}
\end{aligned}
$$

6. The Mahgoub transform of a delta function

$$
M(\delta)=\hat{M}_{\delta}(v)=v
$$

7. Change of Scale by nonzero integer $\alpha$

$$
M(f(\alpha t))=\hat{M}\left(\frac{v}{\alpha}\right)
$$

8. The Mahgoub transform of derivative $F^{\prime}(t), F^{\prime \prime}(t)$

$$
\begin{array}{r}
M\left(F^{\prime}(t)\right)=v(\hat{M}(v)-F(0)) \\
M\left(F^{\prime \prime}(t)\right)=v^{2} \hat{M}(v)-v F^{\prime}(0)-v^{2} F(0)
\end{array}
$$

9. The inverse Mahgoub transform is related with Bromwich contour integral

$$
f(x)=M^{-1}(f(v))=\lim _{v \rightarrow \infty} \frac{1}{2 \pi i} \int_{\gamma-i v}^{\gamma+i v} \hat{M}(f(v))(x) e^{v x} \mathrm{~d} x
$$

\section{Relation between Mahgoub transform and other integral transforms}

i) Connection between Kamal transform and Mahgoub transform :- If $\hat{M}(v)$ and $\hat{K}(v)$ are Mahgoub and Kamal transform of $\mathrm{f}(\mathrm{x})$ then

$$
\hat{K}(v)=v \hat{M}\left(\frac{1}{v}\right)
$$

ii) Mahgoub Laplace Duality :- If $\hat{M}(v)$ and $F(v)$ are the Mahgoub and Laplace transform of $\mathrm{f}(\mathrm{x})$ then

$$
\hat{M}(v)=v F(v)
$$

iii) Similarity Mahgoub-Sumudu Duality is given by

$$
\hat{M}(v)=S\left(\frac{1}{v}\right)
$$

iv) Mahgoub Elzaki Duality is

$$
\hat{M}(v)=v^{2} E(v)
$$

v) Mahgoub Aboodh Duality is

$$
\hat{M}(v)=v^{2} A(v)
$$

\section{Linearity}

If $f(x)$ and $g(x)$ have Mahgoub transform as $M(f)$ and $M(g)$ then

$M(\alpha f(x)+\beta g(x))=\alpha M(f)+\beta M(g) \quad$ where $\quad \alpha \beta \in \mathbb{R}_{+}$.

Proof.

$$
\begin{aligned}
& M(\alpha f(x)+\beta g(x)) \\
& =v \int_{0}^{\infty}(\alpha f(x)+\beta g(x)) \cdot e^{-v x} \mathrm{~d} x \\
& =v \int_{0}^{\infty} \alpha f(x) \cdot e^{-v x} \mathrm{~d} x+v \int_{0}^{\infty} \beta g(x) \cdot e^{-v x} \mathrm{~d} x \\
& =\alpha\left[\int_{0}^{\infty} v f(x) \cdot e^{-v x} \mathrm{~d} x\right]+\beta\left[\int_{0}^{\infty} v g(x) \cdot e^{-v x} \mathrm{~d} x\right] \\
& M(\alpha f(x)+\beta g(x))=\alpha M(f)+\beta M(g)
\end{aligned}
$$

\section{Convolution}

The Convolution of two functions $f$ and $g$ is given by

$$
(f * g)(x)=\int_{0}^{\infty} f(u) g(x-u) \mathrm{d} u
$$

The Convolution theorem of two function $f, g$ of Mahgoub transform is given by,

$$
M(f * g)(v)=\frac{1}{v} M(f) \cdot M(g)
$$

$$
\begin{aligned}
& M(f * g)(v) \\
& =\int_{0}^{\infty}(f * g)(x)\left[v e^{-v x}\right] \mathrm{d} x \\
& =\int_{0}^{\infty} \int_{0}^{\infty} f(u) g(x-u) \mathrm{d} u\left(v e^{-x v}\right) \mathrm{d} x \\
& =\int_{0}^{\infty} f(u) \mathrm{d} u \int_{0}^{\infty} g(x-u) v e^{-x v} \mathrm{~d} x
\end{aligned}
$$


Substitute $\quad x-u=y$, we have

$$
\begin{aligned}
& M(f * g)(v)=\int_{0}^{\infty} f(u) \mathrm{d} u \int_{0}^{\infty} g(y) v e^{-(y+u) v} \mathrm{~d} y \\
& =\int_{0}^{\infty} f(u) \cdot e^{-u v} \mathrm{~d} u \int_{0}^{\infty} g(y) v e^{-y v} \mathrm{~d} y \\
& =\frac{1}{v}\left\{v \int_{0}^{\infty} f(u) e^{-u v} \mathrm{~d} u \cdot v \int_{0}^{\infty} g(y) e^{-y v} \mathrm{~d} y\right\} \\
& =\frac{1}{v} M(f) \cdot M(g) \\
& M(f * g)(v)=\frac{1}{v} M(f) \cdot M(g)
\end{aligned}
$$

\section{Mahgoub Transform of Distribution}

Let $H(\mathbb{R})$ be the space of infinitely smooth function on $\mathbb{R}$ and $H^{\prime}(\mathbb{R})$ be the dual of $H(\mathbb{R})$ i.e. $H^{\prime}(\mathbb{R})$ is dual space of distribution of compact support.

Let $D(\mathbb{R})$ denote the subspace of $H(\mathbb{R})$ of testing function space of compact support and $D^{\prime}(\mathbb{R})$ is its dual consists of Schwartz space of distributions. Now $D \subset H, H^{\prime} \subset D^{\prime}$.

The kernel of Mahgoub transform $v e^{-v x}$ is clearly a member of $H(\mathbb{R})$. Hence we define the generalized Mahgoub transform $\mathrm{M}$ on $H^{\prime}(\mathbb{R})$ by

$$
\begin{aligned}
& M(f(x))(v)=<f(x), v e^{-v x}> \\
& \quad \text { for every distribution } \quad f \in H^{\prime}(\mathbb{R})
\end{aligned}
$$

Theorem 4.1. $M$ is well defined mapping in the space $H(\mathbb{R})$

Proof. Proof is immediate since, $v e^{-v x} \in H(\mathbb{R})$

Theorem 4.2. $M$ is infinitely smooth and

$$
\frac{d^{k}}{d v^{k}} M(f)(v)=<f(x), \frac{d^{k}}{d v^{k}} v e^{-v x}>\quad f \in H^{\prime}(\mathbb{R})
$$

Proof. This theorem can be proved with the help of [[13], Theorem 2.9.1].

Theorem 4.3. The generalized Mahgoub transform M is Linear.

Proof. Let $f, g \in H^{\prime}(\mathbb{R})$ and $\alpha, \beta$ non negative real number then

$$
\begin{aligned}
& M(\alpha f+\beta g)(v) \\
& =<\alpha f+\beta g, v e^{-v x}> \\
& =<\alpha f, v e^{-v x}>+<\beta g, v e^{-v x}> \\
& =\alpha<f, v e^{-v x}>+\beta<g, v e^{-v x}> \\
& =<\alpha M(f)(v)+\beta M(g)(v)>
\end{aligned}
$$

Theorem 4.4. If $f \in H^{\prime}(\mathbb{R})$ and $g(x)= \begin{cases}f(x-\tau) & x \geq \tau \\ 0 & x<\tau\end{cases}$ then

$$
M(g)(u)=e^{-v \tau} M(f)(v)
$$

Proof. Let $f \in H^{\prime}(\mathbb{R})$ anf $\mathrm{g}$ is defined as above then by translation property of distributional Zemanian [14]

$$
\begin{aligned}
M(g)(v) & =<g(x), v e^{-v x}> \\
& =<f(x-\tau), v e^{-v x}> \\
& =<f(x), v e^{-v(x+\tau)}> \\
& =<g(x), v e^{-v x} \cdot e^{-v \tau}> \\
& =e^{-v \tau}<f(x), v e^{-v x}> \\
M(g)(v) & =e^{-v \tau} M(f)(v)
\end{aligned}
$$

Let us define the Convolution of two functions.

Let $f, g \in H^{\prime}(\mathbb{R})$ we define the generalized Convolution between $\mathrm{f}$ and $\mathrm{g}$ by

$$
<(f * g), \psi(x)>=<f(x),<g(t), \psi(t+x)>>
$$

for every $\psi \in H(\mathbb{R})$

Theorem 4.5. Let $f, g \in H^{\prime}(\mathbb{R})$ then

$$
M(f * g)(v)=\frac{M(f) M(g)}{v} .
$$

Proof. By definition of $M$

$$
\begin{aligned}
M(f * g)(v) & =<(f * g), v e^{-v x}> \\
& =<f(x),<g(t), v e^{-v(x+t)}>> \\
& =<f(x),<g(t), v e^{-v x} \cdot e^{-v t}>> \\
& =<f(x), v e^{-v x}><g(t), e^{-v t}> \\
M(f * g)(v) & =\frac{1}{v}<f(x), v e^{-v x}><g(t), v e^{-v t}> \\
M(f * g)(v) & =\frac{1}{v} M(f) \cdot M(g)
\end{aligned}
$$

Theorem 4.6. Let $f \in H^{\prime}(\mathbb{R})$ then the following holds

$$
M(x f(x))(v)=\frac{1}{v}\left[M(f)(v)-\frac{d}{d v} M(f)(v)\right]
$$

Proof. By using the properties of Mahgoub transform and 
Theorem (2.2) we get

$$
\begin{aligned}
& \frac{d}{d v} M(f) \\
& =\frac{d}{d v}<f(x), v e^{-v x}> \\
& =<f(x), \frac{d}{d v} v e^{-v x}> \\
& =<f(x), v e^{-v x}(-x)+e^{-v x}> \\
& =<f(x),-x v e^{-v x}+e^{-v x}> \\
& =<f(x), e^{-v x}>-<f(x), x v e^{-v x}> \\
& =<f(x), e^{-v x}>-<x f(x), v e^{-v x}> \\
& =\frac{1}{v}<f(x), v e^{-v x}>-<x f(x), v e^{-v x}> \\
& =\frac{d}{v} M(f)(v)-M(x f)(v) \\
& M(x f)(v)=\frac{1}{v} M(f)(v)-\frac{d}{d v} M(f)
\end{aligned}
$$

Theorem 4.7 (Shifting Theorem). Let $f \in H^{\prime}(\mathbb{R})$ then

$$
\begin{aligned}
M\left(e^{a x} f(x)\right)(v) & =<e^{a x} f(x), v e^{-v x}> \\
& =<f(x), v e^{-(v-a) x}> \\
& =<f(x), \frac{v}{(1-a / v)} e^{-\left(1-\frac{a}{v}\right) v x}> \\
& =<f(x), \frac{1}{(1-a / v)} \cdot v e^{-(v-a) x}> \\
& =\frac{v}{(v-a)}<f(x), v e^{-(v-a)) x}> \\
& =\frac{v}{(v-a)} M f(v-a)
\end{aligned}
$$

\section{Mahgoub transform of Boehmians}

Distributions or generalized function are the objects that generalize functions. To differentiate functions whose derivative do not exist in classical sense is possible in distributional sense. In 1983 Boehmians are the objects obtained by abstract algebraic construction to generalized distribution [11]. The original construction was motivated by regular operators [8]. Boehmians are defined as equivalence classes of convolution quotients of functions that are subclass of Mikusinski's operator. The most youngest generalization of functions is the theory of Boehmians.

For Linear Space $\mathrm{G}$ and subspace $\mathrm{F}$ of $\mathrm{G}$ assume to all pair (f, $\phi),(\mathrm{g}, \psi)$ of elements, $\mathrm{f}, \mathrm{g} \in \mathrm{G} \quad \phi, \psi \in \mathrm{F}$ the product $\mathrm{f} * \phi, \mathrm{g} * \psi$ such that the following conditions are satisfied.

1. $\phi * \psi \in F$ and $\phi * \psi=\psi * \phi$

2. $(f * \phi) * \psi=f *(\phi * \psi)$

3. $(f+g) * \phi=(f * \phi)+(g * \phi)$
4. $\lambda(f * \phi)=(\lambda f) * \phi=f *(\lambda \phi) \quad \lambda \in \mathbb{R}$

Let $\triangle$ be the family of sequences from $F$ such that for $f, g \in G$

5. If $\left(\varepsilon_{n}\right) \in \triangle$ and $\left(f * \varepsilon_{n}\right)=\left(g * \varepsilon_{n}\right) \mathrm{n}=1,2, \cdots$ then $\mathrm{f}=\mathrm{g}$

6. $\left(\varepsilon_{n}\right),\left(\mu_{n}\right) \in \triangle \Rightarrow\left(\varepsilon_{n} * \mu_{n}\right) \in \triangle$

Elements of $\triangle$ are called $\triangle$ sequences.

Now consider the class $U$ of pairs of sequences defined by $U=\left\{\left(\left(f_{n}\right),\left(\varepsilon_{n}\right)\right):\left(f_{n}\right) \subseteq G^{\mathbb{N}}, \varepsilon_{n} \in \triangle\right\}$ for each $\mathrm{n}$.

The pair $\left(\left(f_{n}\right),\left(\varepsilon_{n}\right) \in U\right.$ is said to be quotient of sequences denoted by $f_{n} / \varepsilon_{n} \chi$ if $f_{n} * \varepsilon_{m}=f_{m} * \varepsilon_{n} \quad$ for $\quad \forall m, n \in \mathbb{N}$.

Two quotients of sequences $f_{n} / \phi_{n}$ and $g_{n} / \psi_{n}$ are equivalent $f_{n} / \phi_{n} \sim g_{n} / \psi_{n}$ if $f_{n} * \psi_{m}=g_{m} * \phi_{n} \quad$ for $\forall m, n \in \mathbb{N}$.

The relation $\sim$ is an equivalence relation on $U$ and hence splits $U$ into equivalence classes. The equivalence class containing $f_{n} / \phi_{n}$ is denoted by $\left[f_{n} / \phi_{n}\right]$. These equivalence classes are called Boehmians and is denoted by $B(G, F, \triangle, *)$

The sum and multiplication by a scalar of two Boehmians can be defined as,

$$
\left[f_{n} / \phi_{n}\right]+\left[g_{n} / \psi_{n}\right]=\left[\left(\left(f_{n} * \psi_{n}\right)+\left(g_{n} * \phi_{n}\right)\right) /\left(\phi_{n} * \psi_{n}\right)\right]
$$

and

$$
\gamma\left[f_{n} / \phi_{n}\right]=\left[\gamma f_{n} / \phi_{n}\right] \quad \gamma \in \mathbb{C}
$$

The operation $*$ and differentiation are given by ,

$$
\left[f_{n} / \phi_{n}\right] *\left[g_{n} / \psi_{n}\right]=\left[\left(f_{n} * g_{n}\right) /\left(\phi_{n} * \psi_{n}\right)\right]
$$

and

$$
D^{\alpha}\left[f_{n} / \phi_{n}\right]=\left[D^{\alpha} f_{n} / \phi_{n}\right]
$$

$\mathrm{G}$ is equipped with notion of convergence.

The intrinsic relationship between the notion of convergence and the product $*$ is given by,

1. If $f_{n} \rightarrow f$ as $n \rightarrow \infty$ in $\mathrm{G}$ and $\phi \in F$ is any fixed element then $f_{n} * \phi \rightarrow f * \phi$ in $\mathrm{G}$ as $n \rightarrow \infty$

2. If $f_{n} \rightarrow f$ as $n \rightarrow \infty$ in G and $\left(\delta_{n}\right) \in \triangle$ then $f_{n} * \delta_{n} \rightarrow f$ in $\mathrm{G}$ as $n \rightarrow \infty$

This operation $*$ can be extended to $B(G, F, \triangle, *) \times F$

3. If $\left[f_{n} / \delta_{n}\right] \in B(G, F, \triangle, *)$ and $\phi \in F$ then $\left[f_{n} / \delta_{n}\right] * \phi=$ $\left[\left(f_{n} * \phi\right) / \delta_{n}\right]$

In $B(G, F, \triangle, *)$ two types of convergences, $\delta$-convergence and $\triangle$-convergence are defined as:

( $\delta$-convergence) A sequence of $\operatorname{Boehmians}\left(\gamma_{n}\right)$ in $B(G, F, \triangle, *)$ is said to be $\delta$ convergent to a Boehmian $\gamma$ in 
$B(G, F, \triangle, *)$ denoted by $\gamma_{n} \stackrel{\delta}{\rightarrow} \gamma$ if there exist a delta sequence $\left(\delta_{n}\right)$ such that

$$
\left(\gamma_{n} * \delta_{n}\right),\left(\gamma * \delta_{n}\right) \in G \quad \forall k, n \in \mathbb{N}
$$

and

$$
\left(\gamma_{n} * \delta_{k}\right) \rightarrow \gamma * \delta_{k} \quad n \rightarrow \infty \quad \text { in } G \quad \text { for every } k \in \mathbb{N}
$$

The following is equivalent statement of $\delta$-convergent. The sequences $\gamma_{n} \stackrel{\delta}{\rightarrow} \gamma(n \rightarrow \infty)$ in $B(G, F, \triangle$,*) if and only if there is $g_{n, k}, g_{k} \in G$ and $\delta_{k} \in \triangle$ such that

$$
\begin{gathered}
\gamma_{n}=\left[g_{n, k} / \delta_{k}\right] \quad \gamma=\left[g_{k} / \delta_{k}\right] \quad \text { and for each } \quad k \in \mathbb{N} \\
g_{n, k} \rightarrow g_{k} \quad \text { as } \quad n \rightarrow \infty \quad \text { in G. }
\end{gathered}
$$

( $\triangle$-convergence) A sequence of Boehmians $\left(\gamma_{n}\right)$ in $B(G, F, \triangle, *)$ is said to be $\triangle$ convergent to a Boehmian $\gamma$ in $B(G, F, \triangle, *)$ denoted by $\gamma_{n} \stackrel{\delta}{\rightarrow} \gamma$ if there exist a $\left(\delta_{n}\right) \in \triangle$ such that

$$
\left(\gamma_{n}-\gamma\right) * \delta_{n} \in G \quad \forall n \in \mathbb{N}
$$

and

$$
\left(\gamma_{n}-\gamma\right) * \delta_{n} \rightarrow 0 \text { as } n \rightarrow \infty \quad \text { in } \quad G .
$$

Now we consider a space of Boehmians $B(H, D, \triangle, *)$ with $H(\mathbb{R})$ as a group $D(\mathbb{R})$ Schwartz space of test functions as a subgroup of $H(\mathbb{R}) . *$ is an operation for $H(\mathbb{R})$ and $D(\mathbb{R})$ and $\triangle$ as set of delta sequence[9]. Also we denote $D^{\prime}(\mathbb{R})$ strong dual of $D(\mathbb{R})$ of Schwartz distribution.

Theorem 5.1. Let $\left[h_{n} / \psi_{n}\right] \in B(H, D, \triangle, *)$ then the sequence $M\left(f_{n}\right)$ converges in $D^{\prime}(\mathbb{R})$ Moreover if $\left[h_{n} / \psi_{n}\right]=\left[g_{n} / \phi_{n}\right]$ in the sense of $B(H, D, \triangle, *)$ then $M\left(f_{n}\right)$ and $M\left(g_{n}\right)$ converge to the same limit.

Proof. For delta sequence $\left(\delta_{n}\right)$ we have,

$$
\begin{aligned}
& M\left(\delta_{n}\right) \rightarrow M(\delta)(v) \\
\Rightarrow M\left(\delta_{n}\right) \rightarrow v & \text { as } \quad n \rightarrow \infty \quad b y(2.6)
\end{aligned}
$$
$k \in \mathbb{N}$.

Let $\phi \in D(\mathbb{R})$ be such that $M\left(\phi_{k}\right)>0$ on the support of $\phi$, $f_{n} / \psi_{n}$ is quotient of sequences implies that $f_{n} * \psi_{m}=$ $f_{m} * \psi_{n}$

$$
\begin{aligned}
M\left(f_{n}\right) \cdot M\left(\psi_{m}\right) & =M\left(f_{m}\right) \cdot M\left(\psi_{n}\right) \\
\text { therefore } M\left(f_{n}\right)(\phi) & =M\left(f_{n}\right) \phi \cdot \frac{M\left(\psi_{k}\right)}{M\left(\psi_{k}\right)} \\
& =M\left(f_{n}\right) \frac{M\left(\psi_{k}\right)}{M\left(\psi_{k}\right)} \cdot \phi \\
& =M\left(f_{n}\right)\left(M\left(\psi_{k}\right)\right) \cdot \frac{\phi}{M\left(\psi_{k}\right)} \\
& =M\left(f_{k}\right)\left(M\left(\psi_{n}\right)\right) \cdot \frac{\phi}{M\left(\psi_{k}\right)} \\
& =M\left(f_{k}\right) \cdot\left(\phi \frac{M\left(\psi_{n}\right)}{M\left(\psi_{k}\right)}\right)
\end{aligned}
$$

$$
\text { Now } \quad\left(\frac{\phi M\left(\psi_{n}\right)}{M\left(\psi_{k}\right)}\right) \rightarrow \frac{\phi \cdot v}{M\left(\psi_{k}\right)} \quad \text { in } \quad D(\mathbb{R})
$$

Hence the sequence $M\left(f_{n}\right)$ converges in $D^{\prime}(\mathbb{R})$

$$
\text { Let }\left[\frac{f_{n}}{\psi_{n}}\right]=\left[\frac{g_{n}}{\phi_{n}}\right] \quad \text { in } \quad B(H, D, \triangle, *) \quad \text { and define }
$$

$$
\begin{gathered}
h_{n}= \begin{cases}f_{\frac{n+1}{2}} * \psi_{\frac{n+1}{2}} & \mathrm{n} \text { is odd } \\
g_{\frac{n}{2}} * \psi_{\frac{n}{2}} & \mathrm{n} \text { is even }\end{cases} \\
\delta_{n}= \begin{cases}\psi_{\frac{n+1}{2}} * \phi_{\frac{n+1}{2}} & \mathrm{n} \text { is odd } \\
\psi_{\frac{n}{2}} * \phi_{\frac{n}{2}} & \mathrm{n} \text { is even }\end{cases} \\
\text { then }\left[\frac{h_{n}}{\delta_{n}}\right]=\left[\frac{f_{n}}{\psi_{n}}\right]=\left[\frac{g_{n}}{\phi_{n}}\right]
\end{gathered}
$$

The sequence $M\left(h_{n}\right)$ converges in $D^{\prime}$ Moreover.

$$
\lim _{n \rightarrow \infty} M\left(h_{2 n-1}\right)=\lim _{n \rightarrow \infty} M\left(f_{n}\right)
$$
limit.

therefore $M\left[\left(h_{n}\right)_{n=1}^{\infty}\right]$ and $M\left(f_{n}\right)$ converge to the same

Similarly $M\left[\left(h_{n}\right)_{n=1}^{\infty}\right]$ and $M\left(g_{n}\right)$ converge to the same limit.

So $\mathrm{M}$ maps $B(H, D, \triangle, *)$ into $D^{\prime}(\mathbb{R})$

We define Mahgoub transform of Boehmians

$$
\begin{aligned}
\gamma=\left[f_{n} / \phi_{n}\right] \in B(H, D, \triangle, *) \quad \text { by } \\
\breve{M}(\gamma)=\lim _{n \rightarrow \infty} M\left(f_{n}\right)
\end{aligned}
$$

Theorem 5.2. The Mahgoub transform $\breve{M}: B(H, D, \triangle, *) \rightarrow$ $D^{\prime}(\mathbb{R})$ is linear.

Proof. Let $\beta_{1}, \beta_{2} \in B(H, D, \triangle, *)$ such that $\beta_{1}=\left[f_{n} / \phi_{n}\right], \beta_{2}=$ $\left[g_{n} / \psi_{n}\right]$ then

$$
\begin{aligned}
\left(\beta_{1}+\beta_{2}\right) & =\left[\left(\left(f_{n} * \psi_{n}\right)+\left(g_{n} * \phi_{n}\right)\right) /\left(\phi_{n} * \psi_{n}\right)\right] \\
\breve{M}\left(\beta_{1}+\beta_{2}\right) & =\lim M\left(f_{n} * \psi_{n}\right)+\lim M\left(g_{n} * \phi_{n}\right) \\
& =\lim _{n \rightarrow \infty} M\left(f_{n}\right)+\lim _{n \rightarrow \infty} M\left(g_{n}\right) \\
& =\breve{M} \beta_{1}+\breve{M} \beta_{2} .
\end{aligned}
$$

Also

$$
\breve{M}(\alpha \beta)=\breve{M}\left(\alpha f_{n} / \phi_{n}\right)=\alpha \lim _{n \rightarrow \infty} M f_{n}=\alpha \breve{M} \beta
$$

where $\alpha \in \mathbb{C}, \beta=\left[f_{n} / \phi_{n}\right]$.

Theorem 5.3. If $\beta=\left[f_{n} / \phi_{n}\right]$ in $B(H, D, \triangle, *)$ and $\breve{M} \beta=0$ in $D^{\prime}$ then $\beta=0$ in $B(H, D, \triangle, *)$. 
Proof. Assume that $\beta=\left[f_{n} / \phi_{n}\right]$ and $\breve{M} \beta=0$, then by using the definition of $\breve{M}$ we get $\lim _{n \rightarrow \infty} M\left(f_{n}\right)=0$ with the help of definition of Mahgoub transform $f_{n} \rightarrow 0$ almost every where in $H^{\prime}(\mathbb{R})$ and $f_{n} / \phi_{n}$ is a zero quotient of functions or equivalently $\beta=\left[f_{n} / \phi_{n}\right]$ is zero equivalence class in $B(H, D, \triangle, *)$.

Theorem 5.4. $\breve{M}$ is one-one mapping from $B(H, D, \triangle$,*) to $D^{\prime}(\mathbb{R})$

Proof. Let $\breve{M} \beta_{1}=\breve{M} \beta_{2}, \beta_{1}, \beta_{2} \in B(H, D, \triangle, *)$ then by above theorem (4.2) and (4.3)

$$
\begin{gathered}
\breve{M}\left(\beta_{1}-\beta_{2}\right)=0 \quad \text { in } \quad D^{\prime}(\mathbb{R}) \\
\beta_{1}-\beta_{2}=0 \quad \Rightarrow \beta_{1}=\beta_{2}
\end{gathered}
$$

Theorem 5.5 (Convolution Theorem). Let $\beta_{1}=\left[f_{n} / \phi_{n}\right], \beta_{2}=$ $\left[g_{n} / \psi_{n}\right] \in \beta$ then we have

$$
\breve{M}\left(\beta_{1} * \beta_{2}\right)=\frac{1}{v} \breve{M}\left(\beta_{1}\right) \cdot \breve{M}\left(\beta_{2}\right) .
$$

Proof.

$$
\begin{aligned}
\breve{M}\left(\left[f_{n} / \phi_{n}\right] *\left[g_{n} / \psi_{n}\right]\right) & =\breve{M}\left[\left(f_{n} * g_{n}\right) /\left(\phi_{n} * \psi_{n}\right)\right] \\
& =\lim _{n \rightarrow \infty} M\left(f_{n} * g_{n}\right) \\
& =\lim _{n \rightarrow \infty} \frac{1}{v} M\left(f_{n}\right) \cdot\left(g_{n}\right) \\
& =\frac{1}{v} \lim _{n \rightarrow \infty} M\left(f_{n}\right) \cdot \lim _{n \rightarrow \infty} M\left(g_{n}\right) \\
& =\frac{1}{v} \breve{M}\left(\beta_{1}\right) \cdot \breve{M}\left(\beta_{2}\right)
\end{aligned}
$$

Theorem 5.6. The Mahgoub transform $\breve{M}(\beta)$ is infinitely smooth.

Proof. Let $\beta=\left[f_{n} / \psi_{n}\right] \in B(H, D, \triangle, *)$ and let $\mathrm{J}$ be bounded set in $\mathbb{R}$ then for $m \in \mathbb{N}$ we have $M\left(\psi_{n}\right)>0$ on $\mathrm{J}$.

$$
\begin{aligned}
\breve{M}(\beta) & =\lim _{n \rightarrow \infty} M\left(f_{n}\right) \\
& =\lim _{n \rightarrow \infty} \frac{M\left(f_{n}\right) \cdot M\left(\psi_{m}\right)}{M\left(\psi_{m}\right)} \\
& =v \lim _{n \rightarrow \infty} \frac{M\left(f_{n} * \psi_{m}\right)}{M\left(\psi_{m}\right)} \\
& =v \lim _{n \rightarrow \infty} \frac{M\left(f_{m} * \psi_{n}\right)}{M\left(\psi_{m}\right)} \\
& =\lim _{n \rightarrow \infty} \frac{M\left(f_{m}\right) \cdot M\left(\psi_{n}\right)}{M\left(\psi_{m}\right)} \\
& =\frac{M\left(f_{m}\right)}{M\left(\psi_{m}\right)} \lim _{n \rightarrow \infty} M\left(\psi_{n}\right) \\
& =\frac{M\left(f_{m}\right)}{M\left(\psi_{m}\right)} \cdot v \quad \text { on } J
\end{aligned}
$$

$$
\text { but } \begin{array}{r}
M\left(f_{n}\right) \cdot M\left(\psi_{m}\right) \in H(\mathbb{R}) \text { and } M\left(\psi_{m}\right)>0 \text { on } \mathbf{J} \\
\Rightarrow \breve{M}(\beta) \quad \text { is infinitely smooth. }
\end{array}
$$

\section{Conclusion}

In this paper we have obtained the relation between Mahgoub transform and other integral transforms. We defined the Mahgoub transform in distributional sense. Also convolution and Boehmian space for Mahgoub transform is defined.

\section{References}

[1] Aggarwal Sudhanshu, Application of Mahgoub transform for Solving population growth and decay problems, Journal of computer and Mathematical Science, 9(10)(2018), 1490-1496.

[2] Aggarwal Sudhanshu, A new Application of Mahgoub transform for Solving Linear ordinary Differential Equation with variable coefficient, Journal of computer and Mathematical Sciences, 9(6)(2018), 520-525.

[3] Aggarwal Sudhanshu, Chauhan Raman, Sharma Nidhi, Solution of Linear Voltera Integro Differential Equation of Second kind using Mahgoub transform, IJLTEMAS, 7(5)(2018), 173-176.

[4] Al-Omari S.K.Q., On the Application of Natural transforms, International Journal of Pure and Applied Mathematics, 85(4)(2013), 729-744.

[5] Al-Omari S.K.Q., Al-Omari J.F., Hartley Transform for $L^{p}$ Boehmians and space of ultra distributions, International Mathematical Forum, 7(2012), 433-443.

[6] Al-Omari S.K.Q. and Kilicman.A., Note on Boehmians for class of optical Fresnel Wavelet Transforms, Journal of Function space and Applications, Vol. 2012, Article ID405368.

[7] Al-omari S.K.Q. and Kilicman.A., On Generalized Hartley-Hilbert and Fourier Hilbert transform, Advances in Difference Equation, 232(2012), 12-25.

[8] Boehme T.K., The support of Mikusinski operators, Trans. Amer. Math. Soc., 176(1973), 319-334.

[9] Hassan Taha N.E., Nuruddeen R.I., Kamal A., Sedeeg H., Dualities between Kamal and Mahgoub Integral Transform and some famous Integral transforms, British Journal of Applied Science and Technology, 20(3)(2017), 1-8.

[10] Khan Z.H. and Khan W.A., Natural transform properties and applications, NUST Journal of Engineering Sciences, 1(2008), 127-133.

[11] Mikusinski P., Convergence of Boehmians, Japan J. Math., 9(1983), 159-179.

[12] Mohand M.A. Mahgoub The new integral transform Mahgoub Transform, Advances in Theoretical and Applied Mathematics, 2(4)(2016), 391-398.

[13] Pathak R.S., Integral Transforms of Generalized Functions and Their Applications, Gordon and Breach Science Publishers, Japan, 1997. 
[14] Zemanian A.H., Generalized Integral Transformation, Interscience Publisher, John Wiley and Sons, New York, 1968.

$\operatorname{ISSN}(\mathrm{P}): 2319-3786$

Malaya Journal of Matematik

$\operatorname{ISSN}(\mathrm{O}): 2321-5666$

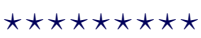

